\title{
Successful Mobile Extracorporeal Membrane Oxygenator for COVID-19 Severe Respiratory Failure.
}

\author{
Nicolas Brozzi ${ }^{1}$, Jaime Hernandez-Montfort ${ }^{1}$, Edward Noguera ${ }^{1}$, Carla McWilliams ${ }^{1}$, \\ Cedric Sheffield ${ }^{1}$, Jeffrey Jacobs ${ }^{1}$, Viviana Navas ${ }^{1}$, Mauricio Velez ${ }^{1}$, Robert Cubeddu ${ }^{1}$, and \\ Jose Navia ${ }^{1}$ \\ ${ }^{1}$ Cleveland Clinic Florida
}

July 7,2020

\begin{abstract}
High volume ECMO centers have developed mobile ECMO programs in recent years to facilitate implementation of ECMO support at hospitals with lower capabilities, and transfer these patients for further care. We report a case of mobile ECMO on patient with COVID-19 related ARDS, and discuss the potential application in current SARS-CoV-2 pandemic.
\end{abstract}

Background: The public health emergency of Coronavirus Disease 2019 (COVID-19) caused by the novel coronavirus SARS-CoV-2 continues to compromise global health and well-being. ${ }^{1}$

Most patients will develop mild flu-like symptoms and quickly recover, but a small subgroup of patients will progress to severe acute respiratory failure, requiring admission to an intensive care unit for increased level of support including mechanical ventilation and ECMO. ${ }^{2}$

Recommendations for ECMO support for patients with severe pulmonary failure related to SARS-CoV2 infection is based on current developing experiences and reports from previous epidemics. ${ }^{3}{ }^{4}$ The World Health Organization (WHO) interim guidelines recommend the use of ECMO to support eligible patients with COVID-19 related acute respiratory distress syndrome (ARDS) in expert centers with sufficient volumes to ensure clinical expertise. The FDA has recently approved the application of ECMO and cardiopulmonary bypass circuits to support these patients. ${ }^{5}$

Mobile ECMO has developed in recent years at major ECMO programs around the world as a way to facilitate implementation of ECMO support at hospitals that lack this service, transfer patients on ECMO support from one hospital with lesser resources to institutions with additional capabilities to provide prolonged ECMO support, or provide additional therapies to facilitate weaning off ECMO, and bridge patients to the next therapy. We report a case of mobile ECMO, and discuss the potential application in current SARS-CoV-2 pandemic.

Clinical Case: A 62 year old patient with history of exposure to COVID-19+ patient during recent travel to England on February $27^{\text {th }}, 2020$ was admitted at a community hospital on March $11^{\text {th }}, 2020$ with symptoms of persistent fever and cough for 5 days accompanied by progressive shortness of breath in the previous 24 hours. The patient rapidly progressed to advanced respiratory failure requiring mechanical ventilator support within 12 hours of admission, and this failure required increased support settings including prone ventilation. All were insufficient to provide adequate oxygenation. Forty-eight hours after admission the patient was placed in the prone position on pressure regulated volume control mechanical ventilation with $100 \% \mathrm{FiO}$, progressively hypoxemic, and in respiratory acidosis $\left(\mathrm{pH}=7.06, \mathrm{pCO}_{2}=82 \mathrm{mmHg}, \mathrm{PaO} 2=\right.$ $59 \mathrm{mmHg}$ ). Upon consultation to our program, the patient was discussed by a multidisciplinary team and 
met clinical inclusion criteria including age $<65$ years, no major chronic comorbidities, and acute single organ dysfunction limited to the lung. Hospital administration approved deployment of our ECMO Team with the goal of instituting ECMO at the referring hospital and transfer of the patient to our institution. Emergency hospital privileges were provided to our cardiothoracic surgeon for implantation of ECMO at the community hospital. Upon arrival to referring hospital, after confirming the patient's clinical condition and laboratory results, final decision was made to proceed with veno-venous ECMO support. The strategy for the procedure was discussed with local SICU healthcare providers. The patient was turned back to the supine position, and quickly prepped and draped, as oxygen saturation declined $<88 \%$. Cannulation was performed by implantation of $25 \mathrm{Fr}$ venous cannula in the right common femoral vein and a $20 \mathrm{Fr}$ arterial cannula in the right internal jugular vein, and both were connected to the corresponding venous and arterial lines of the ECMO circuit. A portable ECMO unit (CardioHelp System, Maquet Vascular Systems, Merrimack, NH USA) was used to establish veno-venous ECMO (VV ECMO) support at $3.5 \mathrm{~L} / \mathrm{min}$ with $100 \%$ oxygen blend, resulting in immediate improvement of blood pressure and pulse oximetry, allowing the weaning of both vasopressor (norepinephrine) and mechanical ventilation. Arterial blood gases confirmed improved gas exchange $\left(\mathrm{pH}=7,32, \mathrm{PCO}_{2}=48 \mathrm{mmHg}, \mathrm{PaO} 2=153 \mathrm{mmHg}\right)$.

Transfer of the patient involved a 60 miles trip via ambulance with 2 crew members, a physician, a nurse, and a perfusionist all wearing personal protective equipment (PPE). Upon arrival, the patient was transferred to a negative pressure room, and the portable ECMO unit was switched to the routinely used ECMO unit (Rotaflow Console, Maquet Cardiopulmonary AG, Rastatt, Germany).

The patient remained stable on VV ECMO and mechanical ventilation. He remained with single organ dysfunction limited to the lungs, progressively improved CXR and clinical examination, which lead to weaning of ECMO at day 21, and extubation on day 25. Antiviral therapy included a 5-day course of hydroxychloroquine and azithromycin, followed by 10-day course of remdesivir. The patient developed pseudomonas pneumonia, requiring 10 day course of cefepime. He continued to improve clinically with two negative COVID-19 PCR results at day 30 and 31 of hospital admission, and was discharged from the hospital 5 days later, completing 10 days of IV cefepime for treatment of pseudomona aeruginosa pneumonia.

Discussion: SARS-CoV-2 infection has quickly developed into a pandemic affecting the population of over 200 countries. Health systems around the globe are overwhelmed by an unprecedented number of patients with advanced respiratory failure. The role of ECMO for selective patients with advanced respiratory failure related to COVID-19 has been recognized, but it is a highly specialized and resource intensive therapy. International societies recommend provision of ECMO support by specialized centers. ${ }^{6}$

Most experienced centers around the world have revised indications of ECMO for patients presenting with COVID-19 related respiratory failure, including more stringent inclusion criteria related to age ( $<65$ years) and the absence of chronic comorbidities or chronic organ dysfunction. ECMO programs are also limiting mobile ECMO services in an effort to decrease the risks to healthcare personnel and preserve limited resources. Preliminary observations indicate that approximately $1 \%$ of patients hospitalized for COVID-19 might benefit from ECMO support, and these patients would be best treated at experienced centers with a multidisciplinary approach as illustrated by this clinical case. ${ }^{7}$ Concerns related to exposure of healthcare providers might be lower than expected. These patients present to the ECMO team intubated with airway connected to a close ventilator circuit and ECMO provides the clinical stability to facilitate their transfer. Nonetheless, all personnel involved in mobile ECMO operations should adhere to strict protocols related to use of PPE, and the vehicle and devices used for the transfer of the patient should undergo terminal decontamination. ${ }^{8}$ We believe high volume ECMO centers should reconsider restrictive mobile ECMO policies. The establishment of regionalized mobile ECMO centers would need to involve coordination among area hospitals with regard to communication, reciprocity considerations, and bioethics teams. Development of these regional hubs of ECMO expertise with mobile capabilities could offer needed rescue support to patients and provide ideal healthcare services to patients within specific regions.

Figure 1: Multiple dimensions included in treatment of patient with COVID-19 related ARDS. 
References:

1. WHO. World Health Organization Emergency COVID-19 Dashboard. https://extranet.who.int/publicemergency. Accessed on 4/8/2020.

2. MacLaren G, Fisher D, Brodie D. Preparing for the most critically ill patients with COVID-19: the potential role of extracorporeal membrane oxygenation. JAMA 2020; published online Feb 19. DOI:10.1001/jama.2020.2342.

3. Davies A, Jones D, Bailey M, et al. Extracorporeal membrane oxygenation for 2009 Influenza A(H1N1) acute respiratory distress syndrome. JAMA 2009; 302: 1888-95.

4. Arabi YM, Al-Omari A, Mandourah Y, et al. Critically ill patients with the Middle East respiratory syndrome: a multicenter retrospective cohort study. Crit Care Med 2017; 45: 1683-95.

5. Enforcement Policy for Extracorporeal Membrane Oxygenation and Cardiopulmonary Bypass Devices during the Coronavirus Disease 2019 (COVID-19) Public Health Emergency. https://www.fda.gov/regulatory-information/search-fda-guidance-documents/enforcementpolicy-extracorporeal-membrane-oxygenation-and-cardiopulmonary-bypass-devices-during?utm_campaign=2020-04-06\%20COVID-19\%20ECMO\%20Therapy\%20Devices\&utm_medium=email\&utm_source=Eloqua. Accessed on April 8 ${ }^{\text {th }}, 2020$.

6. Ramanathan K, Antognini D, Combes A, et al. Planning and provision of ECMO services for severe ARDS during the COVID-19 pandemic and other outbreaks of emerging infectious diseases. Lancet Respir Med. 2020. Published online March 20, 2020. https://doi.org/10.1016/52213-2600(20)30121-1.

7. "Extracorporeal Life Support Organization COVID-19 Interim Guidelines: A consensus document from an international group of interdisciplinary ECMO providers". https://www.elso.org/COVID19.aspx Accessed April 9th, 2020.

8. Interim Infection Prevention and Control Recommendations for Patients with Suspected or Confirmed Coronavirus Disease 2019 (COVID-19) in Healthcare Settings. https://www.cdc.gov/coronavirus/2019-ncov/infection-control/control-recommendations.html. Accessed April $9^{\text {th }}, 2020$.
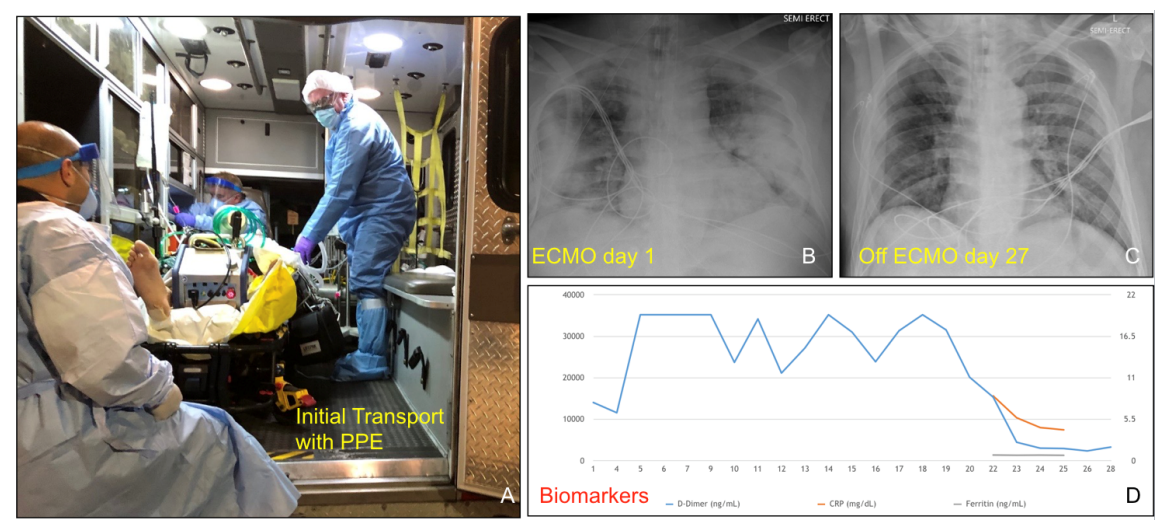